CHIBA-EP-57

revised, September 1992

\title{
Treatment of Constraints in Stochastic Quantization Method and Covariantized Langevin Equation
}

\author{
KenJI IKEGAMI \\ Graduate school of Science and Technology, \\ Chiba University, \\ 1-33 Yayoi-cho, Inageku, Chiba 263, Japan \\ Tadahiko Kimura and Riuji Mochizuki \\ Department of Physics, Faculty of Science, \\ Chiba University, \\ 1-33 Yayoi-cho, Inageku, Chiba 263, Japan
}

\begin{abstract}
We study the treatment of the constraints in stochastic quantization method. We improve the treatment of the stochastic consistency condition proposed by Namiki et al. by suitably taking account of the Ito calculus. Then we obtain an improved Langevin equation and the Fokker-Planck equation which naturally leads to the correct path integral quantization of the constrained system as the stochastic equilibrium state. This treatment is applied to $O(N)$ non-linear $\sigma$ model and it is shown that singular terms appearing in the improved Langevin equation cancel out the $\delta^{n}(0)$ divergences in one loop order. We also ascertain that the above Langevin equation, rewritten in terms of independent variablesis, actually equivalent to the one in the general-coordinate-transformation-covariant and vielbein-rotationinvariant formalism.
\end{abstract}

* To be published in Nucl. Phys. B 


\section{1. INTRODUCTION}

Stochastic quantization method (SQM) was first proposed by Parisi and Wu. ${ }^{[12]}$ They showed that the method could be applied to gauge theory without the gauge fixing procedure. That is, in SQM it is not necessary to introduce the Faddeev-Popov ghost fields. Nevertheless the method produces the same contributions as those due to ghost fields, which was perturbatively confirmed first for Yang-Mills field ${ }^{[3]}$ and recently for non-Abelian anti-symmetric tensor field. ${ }^{[4]}$

How to handle the constrained system in SQM was discussed by Namiki et al. in ref.5. They constructed Langevin equation for the system under the holonomic constraints by imposing the stochastic consistency condition. In the path integral quantization method the constraints introduce a determinant factor into path integral measure, which requires that in SQM Langevin equation for the constrained system is constructed so that the equilibrium Fokker-Planck distribution derived from the Langevin equation has the same determinant factor. They showed that the equilibrium distribution coincided with the path integral distribution. Nevertheless they had to use the 5-dimensional stochastic path integral representation of the transition probability distribution of stochastic process and could not derive FokkerPlanck equation directly from Langevin equation, because they did not take account of Ito calculus ${ }^{[6]}$ in their treatment of stochastic consistency condition. One of the main purposes of this paper is to improve Langevin equation of ref.5 by suitably taking account of Ito calculus, and to show that the improved Langevin equation leads to the Fokker-Planck equation which directly gives the correct path integral representation as the equilibrium distribution. Our improvement introduces some singular terms proportional to $\delta(0)$ in the Langevin equation. So, the Langevin equation of ref.5 is correct in the dimensional regularization scheme, but not in other regularization schemes and the general path integral distribution with constraints cannot be directly obtained as the equilibrium Fokker-Planck distribution.

On the other hand, if the constraints are solved explicitly and the system is described in terms of the independent variables only, the action of the system proves to have generally field-dependent metric. In this case we must apply the generalcoordinate-transformation (GCT)-covariant and vielbein-rotation (VR)-invariant 
Langevin equation to the system. ${ }^{[7]}$ It was not clear whether the latter Langevin equation is equivalent to the above Langevin equation for the constrained system. This point will be clarified in this paper.

Next we discuss about $\mathrm{O}(\mathrm{N})$ non-linear $\sigma$ model as an example of such con-

strained system. The model was studied by many authors. ${ }^{[9]}$ We apply the above two methods to the model and clarify the role of singular terms introduced into the Langevin equation with the stochastic perturbation theory. It will be shown that in both these methods these singular terms are necessary to cancell the $\delta^{n}(0)$ divergences appearing in one-loop expansion.

This paper is organized as follows. In section 2 we study the improved treatment of the constraints in SQM and show directly that the equilibrium Fokker-Planck distribution coincides with the path integral distribution. In section 3 it is shown that the improved Langevin equation for the constrained system is equivalent to the GCT-covariant and VR-invariant Langevin equation. In section 4 we apply both the improved Langevin equation for the constrained system and the GCT-covariant and VR-invariant Langevin equation to $\mathrm{O}(\mathrm{N})$ non-linear $\sigma$ model and examine the cancellation of $\delta^{n}(0)$ divergences. In section 5 we give conclusion and summary. In Appendix, we ascertain that an assumption, which is introduced in section 3, is satisfied at least in $\mathrm{O}(\mathrm{N})$ non-linear $\sigma$ model.

\section{2.CONSTRAined SYSTEM IN SQM}

In this paper we consider the system with variables $q_{i}(x)(i=1,2, \cdots, N)$, regular Lagrangian $L\left(q_{i}, \partial_{\mu} q_{i}\right)(\mu=1,2, \cdots, n)$ and a set of constraints

$$
F_{a}\left(q_{i}\right)=0, \quad(a=1,2, \cdots, M ; N>M) .
$$

In the path integral quantization method the transition amplitude is given by ${ }^{[5][10]}$

$$
\begin{gathered}
\langle f \mid i\rangle=\int D q D J \sqrt{\operatorname{det} D_{a b}} \exp \left[-\int d^{n} x\left(L(q, \partial q)-J_{a} F_{a}\right)\right] . \\
D_{a b} \equiv \frac{\partial F_{a}}{\partial q_{i}} \frac{\partial F_{b}}{\partial q_{i}} .
\end{gathered}
$$


Following the method "time by time constraint" proposed in ref.5, we quantize the above singular system in SQM. The treatment of the stochastic consistency condition is improved by taking account of Ito calculus. It will be shown that the improvement is essential for the acquisition of the Fokker-Planck equation which directly leads to the path integral representation as the equilibrium state.

For the above system, Langevin equation is ${ }^{[5]}$

$$
\begin{gathered}
d q_{i}(t) \equiv q_{i}(t+d t)-q_{i}(t)=-\frac{\delta S}{\delta q_{i}} d t-\frac{\partial F_{a}}{\partial q_{i}} \lambda_{a} d t+d W_{i}, \\
S \equiv \int d^{n} x L
\end{gathered}
$$

where $\lambda_{a}$ is Lagrange multiplier and $d W_{i}$ is defined as

$$
\begin{gathered}
d W_{i}(t) \equiv W_{i}(t+d t)-W_{i}(t), \\
W_{i}(t) \equiv \int^{t} d t^{\prime} \eta_{i}\left(t^{\prime}\right), \\
\left\langle d W_{i}(x, t) d W_{j}\left(x^{\prime}, t\right)\right\rangle=2 \delta_{i j} \delta\left(x-x^{\prime}\right) d t, \\
\left\langle\eta_{i}(x, t) \eta_{j}\left(x^{\prime}, t^{\prime}\right)\right\rangle=2 \delta_{i j} \delta^{n}\left(x-x^{\prime}\right) \delta\left(t-t^{\prime}\right) .
\end{gathered}
$$

and called Wiener process. From (2.8) we may regard $d W$ as order $\sqrt{d t}$. Lagrange multiplier $\lambda_{a}$ is determined by the stochastic consistency condition ${ }^{[5]}$

$$
\dot{F}_{a}(q(t))=0,
$$

where dot denotes fictitious-time derivative. Besides, we demand the initial condition

$$
F_{a}\left(q\left(t=t_{0}\right)\right)=0,
$$

in order to have $F=0$ at any $t$. In SQM, for fictitious-time derivative of any function 
$f(q)^{[6][1]]}$

$$
\frac{d}{d t} f(q(t)) \neq \frac{\partial f}{\partial q} \dot{q}
$$

unless we use Stratonovich calculus. ${ }^{[12]}$ In ref. 5 the above fact is not taken into account. (Their Langevin equation is correct only in the dimensional regularization scheme. In other regularization schemes, the Langevin equation is incorrect and the FokkerPlanck distribution does not coincide with the path integral distribution.) If $\lambda d t$ in eq.(2.4) does not contain terms of order $\sqrt{d t}, d q=d W$ to order $\sqrt{d t}$. From (2.4) we get

$$
d F_{a}=\frac{\partial F_{a}}{\partial q_{i}}\left\{-\left(\frac{\delta S}{\delta q_{i}}+\lambda_{a} \frac{\partial F_{a}}{\partial q_{i}}\right) d t+d W_{i}\right\}+\frac{1}{2}(1-2 b) \frac{\partial^{2} F_{a}}{\partial q_{i} \partial q_{j}} d W_{i} d W_{j}+O\left((\sqrt{d t})^{3}\right),
$$

to order $d t$. Here we use the generalized Ito formula ${ }^{[8]}$ and the product of Wiener process $d W$ and any function $f(q)$ is defined as

$$
\begin{gathered}
\{f(q) d W\}(t) \equiv\{b f(q(t+d t))+(1-b) f(q(t))\} d W(t), \\
0 \leq b \leq 1,
\end{gathered}
$$

where $b=\frac{1}{2}$ corresponds to Stratonovich calculus and $b=0$ to Ito calculus. Requiring $d F_{a}=0$, we get

$$
\begin{gathered}
\lambda_{a} d t=D_{a b}^{-1}\left\{\frac{\partial F_{b}}{\partial q_{i}}\left(-\frac{\delta S}{\delta q_{i}} d t+d W_{i}\right)+\frac{1}{2}(1-2 b) \frac{\partial^{2} F_{a}}{\partial q_{i} \partial q_{j}} d W_{i} d W_{j}\right\}, \\
D_{a b} \equiv \frac{\partial F_{a}}{\partial q_{i}} \frac{\partial F_{b}}{\partial q_{i}} .
\end{gathered}
$$

Here $\lambda_{a} d t$ contains $D_{a b}^{-1} \frac{\partial F_{b}}{\partial q_{i}} d W_{i}$ of order $\sqrt{d t}$, which is inconsistent with the above assumption that $\lambda_{a} d t$ does not contain terms of order $\sqrt{d t}$. So we assume alternatively that the $\lambda_{a} d t$ term contains terms of order $\sqrt{d t}$ like eq.(2.15). Then, from 


$$
\begin{aligned}
d q_{i} & =d W_{i}-\frac{\partial F_{a}}{\partial q_{i}} \lambda_{a} d t \\
& =d W_{i}-\frac{\partial F_{a}}{\partial q_{i}} D_{a b}^{-1} \frac{\partial F_{b}}{\partial q_{j}} d W_{j},
\end{aligned}
$$

to order $\sqrt{d t}$. From (2.17) the modified expression of $d F$ is

$$
\begin{gathered}
d F_{a}=\frac{\partial F_{a}}{\partial q_{i}}\left\{\left(-\frac{\delta S}{\delta q_{i}}-\lambda_{b} \frac{\partial F_{b}}{\partial q_{i}}\right) d t+d W_{i}\right\}+\frac{1}{2}(1-2 b) \frac{\partial^{2} F_{a}}{\partial q_{i} \partial q_{j}} K_{i k} d W_{k} K_{j l} d W_{l}, \\
K_{i j} \equiv \delta_{i j}-R_{i j}, \quad R_{i j} \equiv \frac{\partial F_{a}}{\partial q_{i}} D_{a b}^{-1} \frac{\partial F_{b}}{\partial q_{j}}
\end{gathered}
$$

to order $d t$. Here $R_{i j}, K_{i j}$ are projection operators, vertical each other. The final term in RHS of $\left(2.13^{\prime}\right)$ does not exist in ref.5. From the consistency condition (2.10) the correct expression of $\lambda d t$ becomes

$$
\lambda_{a} d t=D_{a b}^{-1}\left\{\frac{\partial F_{b}}{\partial q_{i}}\left(-\frac{\delta S}{\delta q_{i}} d t+d W_{i}\right)+\frac{1}{2}(1-2 b) \frac{\partial^{2} F_{b}}{\partial q_{i} \partial q_{j}} K_{i k} d W_{k} K_{j l} d W_{l}\right\} .
$$

The above expression is surely correct, because, with the help of $\left(2.15^{\prime}\right), d q$ has the same terms as (2.17) to order $\sqrt{d t}$. From $(2.8)$ and $\left(2.15^{\prime}\right)$, Langevin equation (2.4) becomes

$$
\dot{q}_{i}=K_{i j}\left(-\frac{\delta S}{\delta q_{j}}+\eta_{j}\right)-(1-2 b) \frac{\partial F_{a}}{\partial q_{i}} D_{a b}^{-1} \frac{\partial^{2} F_{b}}{\partial q_{k} \partial q_{l}} K_{k l} \delta^{n}(0)
$$

If we multiply (2.19) by $\frac{\delta F_{a}}{\delta q_{i}}$, we obtain the expression $\left(2.13^{\prime}\right)$ or (2.10). Therefore, the consistency condition (2.10) is embedded in eq.(2.19) and eq.(2.19) means $N$ $M$ independent differential equations. In ref.5 Langevin equation did not have the singular term in (2.19). The same equilibrium Fokker-Planck distribution as (2.2) could not be derived directly from the Langevin equation of ref.5, while, due to the singular term, we can derive the correct Fokker-Planck equation directly from Langevin equation (2.19) as shown below. 
In order to construct the Fokker-Planck equation, we introduce the expectation value of fictitious-time derivative of arbitrary function $g(q)$

$$
\langle\dot{g}(q(t))\rangle \equiv \int D q g(q) \dot{P}(q, t)
$$

where $P(q, t)$ is the transition probability distribution. Using integral by parts and generalized Ito formula, we obtain the Fokker-Planck equation

$$
\dot{P}(q, t)=\frac{\delta}{\delta q_{i}} K_{i j}\left\{\frac{\delta S}{\delta q_{j}}-\frac{\partial^{2} F_{a}}{\partial q_{j} \partial q_{l}} D_{a b}^{-1} \frac{\partial F_{b}}{\partial q_{l}} \delta^{n}(0)+\frac{\delta}{\delta q_{j}}\right\} P(q, t)
$$

due to the singular term in (2.19). Eq.(2.21) cannot be derived from the Langevin equation of ref.5. The probability distribution can include any function $f\left(F_{a}\right)$ because $\frac{\delta F_{a}}{\delta q_{i}}$ is vertical to the projection operater $K_{i j}$. In the equilibrium limit $t \rightarrow \infty$, the probability distibution must satisfy $\dot{P}=0$. In the limit the equation has a solution

$$
P(q)=\int D v_{a} \sqrt{\operatorname{det} D_{a b}} \exp \left(-S-\int d^{n} x v_{a} F_{a}\right)
$$

where we chose $\int D \exp \left(-v_{a} F_{a}\right)$ as $f\left(F_{a}\right)$ in accordance with the initial condition (2.11) in Minkowski space. The above equilibrium probability distribution coincides with eq. (2.2). However, it is strange that the Langevin equation has divergent term of $\delta^{n}(0)$. In section 4 we examine $\mathrm{O}(\mathrm{N})$ non-linear $\sigma$ model as an example of the system under constraint (2.1). In the same example we also show perturbatively that the term proportional to $\delta^{n}(0)$ in eq.(2.19) is needed.

\section{3.EqUiVALENCE TO GCT-COVARIANT}

\section{....... AND VR-INVARIANT LANGEVIN EQUATION}

In this section we shall show that the improved Langevin equation (2.19) is actually equivalent to the Langevin equation in the GCT-covariant and VR-invariant formalism where the constraint (2.1) is explicitly solved and the equation is expressed in terms of independent variables only. 
In general the system may have field-dependent metric (or kernel) $G_{A B}(q)$ after the constraint is solved. According to ref.7, a system with field-dependent metric is described by the GCT-covariant and VR-invariant Langevin equation

$$
d q^{A}=X^{A} d t+E_{m}^{A} d W^{m}
$$

where

$$
\begin{aligned}
& X^{A} \equiv-G^{A B} \frac{\delta S}{\delta q^{B}}+\frac{1}{\sqrt{G}} \frac{\delta}{\delta q^{B}}\left(\sqrt{G} G^{A B}\right)-2 b \frac{\delta E_{m}{ }^{A}}{\delta q^{B}} E_{n}{ }^{B} \delta^{m n} \\
& G^{A B}=E_{m}{ }^{A} E_{n}{ }^{B} \delta^{m n}, \quad A, B, m, n=(1,2, \cdots, N-M ; x) .
\end{aligned}
$$

$G^{A B}$ is the inverse of metric $G_{A B}$, the summation with respect to B includes spacetime integration and $d W^{m}$ is Wiener process defined in section 2. GCT-covariance and VR-invariance mean that Langevin equation is transformed covariantly under general coordinate transformation $q \rightarrow q^{\prime}=f(q)$ and is invariant under vielbein rotation $E_{m}^{A} \rightarrow E_{n}^{A} \Lambda_{m}^{n}$. $d q^{A}$ and $E_{m}^{A} d W^{m}$ are not transformed covariantly and two extra terms in (3.2) are required to be GCT-covariant and VR-invariant.

In order to decompose variables into constraint variables and independent ones, we introduce a new set of variables $\left\{Q^{\mu}\right\}(\mu=1,2, \cdots, N) \cdot{ }^{[5]} Q^{\mu}$ 's are expressed in terms of $q_{i}$ 's $(i=1,2, \cdots, N)$ as

$$
\delta Q^{\mu}=e_{i}^{\mu} \delta q_{i}, \quad \text { or } \quad \frac{\partial Q^{\mu}}{\partial q_{i}}=e_{i}^{\mu}
$$

where $e_{i}^{\mu}$ is vielbein field defined as follows. First $e_{i}^{a}$ and $e_{a, i}(a=N-M+1, \cdots, N)$ are defined as

$$
e_{i}^{a}=\frac{\partial F_{a}}{\partial q_{i}}, \quad e_{a, i}=D_{a b}^{-1} \frac{\partial F_{b}}{\partial q_{i}}
$$

i.e. $Q^{a}=F_{a}$. Then, $e^{A} i(A=1,2, \cdots, N-M)$ is chosen so as to satisfy

$$
e_{i}^{A} e_{a, i}=0
$$


and its inverse $e_{A, i}$ is defined as

$$
e_{A, i}=\left(g^{-1}\right)_{A B} e_{i}^{B}, \quad g^{A B} \equiv e_{i}^{A} e_{i}^{B},
$$

where we assume that $g^{A B}$ is non-singular. From the above definition it turns out that $e_{i}^{\mu}$ and $e_{\mu, i}$ satisfy the following relations

$$
\begin{gathered}
e_{i}^{\mu} e_{\nu, i}=\delta_{\nu}^{\mu}, \quad e_{i}^{\mu} e_{\mu, j}=\delta_{i j}, \\
e_{i}^{A} e_{i}^{a}=e_{i}^{A} e_{a, i}=e_{A, i} e_{i}^{a}=e_{A, i} e_{a, i}=0, \\
e_{i}^{A} e_{A, j}=K_{i j}, \quad e_{i}^{a} e_{a, j}=R_{i j}, \\
K_{i j} e_{j}^{a}=K_{i j} e_{a, j}=0, \quad R_{i j} e_{j}^{A}=R_{i j} e_{A, j}=0 . \\
\operatorname{det}\left(e_{i}^{\mu}\right) \neq 0 .
\end{gathered}
$$

From (3.11) the manifold spanned by $q_{i}$ 's is identical with the one by $Q^{\mu}$ 's. With the help of the same discussion as made about eq. $\left(2.13^{\prime}\right), d Q^{\mu}$ is written as follows

$$
\begin{aligned}
d Q^{\mu} \equiv Q^{\mu}(t+d t)-Q^{\mu}(t) & =\frac{\partial Q^{\mu}}{\partial q_{i}} d q_{i}+(1-2 b) \frac{\partial^{2} Q^{\mu}}{\partial q_{i} \partial q_{j}} K_{i j} d t \delta^{n}(0) \\
& =e^{\mu}{ }_{i} d q_{i}+(1-2 b) \frac{\partial e^{\mu}}{\partial q_{j}} e^{A} i e_{A, j} d t \delta^{n}(0) .
\end{aligned}
$$

Then, from $\left(2.13^{\prime}\right)$ constraint variables $Q^{a}$ 's satisfy Langevin equation

$$
\begin{aligned}
& d Q^{a}=e_{i}^{a}\left\{K_{i j}\left(-\frac{\delta S}{\delta q_{j}} d t+d W_{j}\right)-(1-2 b) \frac{\partial F_{a}}{\partial q_{i}} D_{a b}^{-1} \frac{\partial^{2} F_{b}}{\partial q_{j} \partial q_{l}} K_{j l} d t \delta^{n}(0)\right\} \\
& +(1-2 b) \frac{\partial e_{i}^{a}}{\partial q_{j}} e_{i}^{A} e_{A, j} d t \delta^{n}(0), \\
& =0 \text {, }
\end{aligned}
$$

to order $d t$. From (3.13) and the initial condition (2.11), constraint variables $Q^{a}$ 's 
are zero for all $t$. As for the independent variables $Q^{A}$ 's we get

$$
\begin{aligned}
& d Q^{A}= e_{i}^{A}\left\{K_{i j}\left(-\frac{\delta S}{\delta q_{j}} d t+d W_{j}\right)-(1-2 b) \frac{\partial F_{a}}{\partial q_{i}} D_{a b}^{-1} \frac{\partial^{2} F_{b}}{\partial q_{j} \partial q_{l}} K_{j l} d t \delta^{n}(0)\right\} \\
&+(1-2 b) \frac{\partial e_{i}^{A}}{\partial q_{j}} e_{i}^{B} e_{B, j} d t \delta^{n}(0), \\
&=-g^{A B} \frac{\delta S}{\delta Q^{B}} d t+(1-2 b) \frac{\partial e_{i}^{A}}{\partial Q^{B}} e^{B} d t \delta^{n}(0)+e_{i}^{A} d W^{i} .
\end{aligned}
$$

The above Langevin equation is not invariant under vielbein rotation $e_{i}^{A} \rightarrow e^{A}{ }_{j} \Lambda_{i}^{j}(Q)$ because Wiener process $d W^{i}$ is defined in a manifold spanned by original variables $q_{i}$ 's and we must not consider the rotation in the manifold. If we perform fielddependent rotation in the manifold, $\Lambda_{j}^{i} d W^{j}$ is not Wiener process, i.e. $\left\langle\Lambda^{i}{ }_{j} d W^{j}\right\rangle \neq 0$. In order to reduce (3.14) to the form of (3.1) we decompose the vielbein as follows:

$$
\begin{gathered}
e_{i}^{A}=E_{I}^{A}(Q) \epsilon_{i}^{I}(Q), \quad I=(1,2, \cdots, N-M), \\
\epsilon_{i}^{I} \epsilon_{i}^{J}=\delta^{I J}, \quad e_{i}^{A} e_{i}^{B}=E_{I}^{A} E_{J}^{B} \delta^{I J}=g^{A B},
\end{gathered}
$$

and define $d W^{I}$ by

$$
d W^{I} \equiv \epsilon_{i}^{I} d W^{i}-2 b \frac{\partial \epsilon_{i}^{I}}{\partial Q^{B}} E^{B}{ }_{J} \epsilon_{i}^{J} \delta^{n}(0)
$$

Then, we obtain

$$
\left\langle d W^{I}\right\rangle=0, \quad\left\langle d W^{I}(x) d W^{J}(y)\right\rangle=2 \delta^{I J} d t \delta^{n}(x-y) .
$$

$d W^{I}$ is desirable Wiener process and with $d W^{I}$ Langevin equation is written as

$$
d Q^{A}=-g^{A B} \frac{\delta S}{\delta Q^{B}} d t+\frac{\partial}{\partial Q^{B}}\left(E_{I}^{A} \epsilon_{i}^{I}\right) E_{J}^{B} \epsilon_{i}^{J} d t \delta^{n}(0)-2 b \frac{\partial E_{I}^{A}}{\partial Q^{B}} E_{J}^{B} \delta^{I J} d t \delta^{n}(0)+E_{I}^{A} d W^{I}
$$

From now on we regard $E_{I}^{A}$ as vielbein corresponding to $E_{m}^{A}$ in (3.1). In fact the 
above Langevin equation is invariant under vielbein rotation

$$
E_{I}^{A} \rightarrow E_{J}^{A} \Lambda_{I}^{J}
$$

Langevin equation (3.19) is a little different from GCT-covariant and VR-invariant Langevin equation (3.1). Here we assume

$$
e_{i}^{A} \nabla_{B} e_{i}^{B} \equiv \frac{1}{\sqrt{g}} \frac{\partial}{\partial Q^{B}}\left(\sqrt{g} e_{i}^{B}\right) e_{i}^{A}=0
$$

where $\nabla_{B}$ is covariant derivative in Riemanian manifold spanned by $Q^{A}$. Eq.(3.21) is usually presumed, because $g^{A B}$ satisfies the metric condition $\nabla_{A} g^{B C}=0$ and the latter condition makes eq.(3.21) naturally understandable. We ascertain in Appendix that the above assumption is satisfied in $\mathrm{O}(\mathrm{N})$ non-linear $\sigma$ model. With (3.21), Langevin equation (3.19) is reduced to

$$
\begin{gathered}
d Q^{A}=-g^{A B} \frac{\delta S}{\delta Q^{B}} d t+\frac{1}{\sqrt{g}} \frac{\partial}{\partial Q^{B}}\left(\sqrt{g} g^{A B}\right) \delta^{n}(0) d t-2 b \frac{\partial E_{I}^{A}}{\partial Q^{B}} E_{J}^{B} \delta^{I J} \delta^{n}(0) d t+E_{I}^{A} d W^{I} \\
g \equiv \operatorname{det}\left(g_{A B}\right)
\end{gathered}
$$

Covariant derivative in (3.21) does not include spin connection because, as mentioned above, the rotation must not be considered in the manifold spanned by $q_{i}$ 's. Eq.(3.22) is equivalent to GCT-covariant and VR-invariant Langevin equation (3.1). Thus it is ascertained that Langevin equation (2.19) for the constrained system is equivalent to GCT-covariant and VR-invariant Langevin equation (3.1). That Langevin equation also has divergent term including $\delta^{n}(0)$ and, in the next section, we apply the Langevin equation to $\mathrm{O}(\mathrm{N})$ non-linear $\sigma$ model and see the cancellation of $\delta^{n}(0)$ divergences. 


\section{4. $\mathrm{O}(\mathrm{N})$ NON-LINEAR $\sigma$ MODEL}

$\mathrm{O}(\mathrm{N})$ non-linear $\sigma$ model is defined by action

$$
S=\frac{1}{2} \int d^{n} x \partial_{\mu} \Phi_{i} \partial_{\mu} \Phi_{i}, \quad(\mu=1,2, \cdots, n ; i=1,2, \cdots, N)
$$

and constraint

$$
F=\Phi_{i} \Phi_{i}-\frac{1}{\alpha}=0
$$

where $\alpha$ is constant. The constraint is an example of (2.1). Applying Langevin equation (2.19) to the model, we obtain

$$
\dot{\Phi}_{i}=\left(\delta_{i j}-\alpha \Phi_{i} \Phi_{j}\right)\left(\partial^{2} \Phi_{j}+\eta_{j}\right)-(1-2 b) \alpha(N-1) \Phi_{i} \delta^{n}(0)
$$

The above equation includes (2.19)-type constraint (4.2) and means $N$-1 independent equations. From eq.(4.2) $\Phi_{i}$ has non-zero vacuum expectation value. We shift the field

$$
\begin{gathered}
\phi_{i} \equiv \Phi_{i}-<\Phi_{i}>, \\
v_{i} \equiv \frac{1}{\sqrt{\alpha}}<\Phi_{i}>, \quad v_{i} v_{i}=1,
\end{gathered}
$$

and with the shifted field $\phi$ Langevin equation is written

$$
\begin{gathered}
\dot{\phi}_{i}=\left\{K_{i j}-\sqrt{\alpha}\left(v_{i} \phi_{j}+v_{j} \phi_{i}\right)-\alpha \phi_{i} \phi_{j}\right\}\left\{\partial^{2} \phi_{j}+\eta_{j}\right\} \\
-(1-2 b) \alpha(N-1)\left(\frac{1}{\sqrt{\alpha}} v_{i}+\phi_{i}\right) \delta^{n}(0) \\
K_{i j} \equiv \delta_{i j}-v_{i} v_{j} .
\end{gathered}
$$

Going to momentum space and integrating eq.(4.6) with respect to $t$, we get 


$$
\begin{aligned}
\phi_{i}(k, t)= & \int^{t} d \tau G_{i j}(k, t-\tau)\left[K_{j l} \eta_{l}(k, \tau)+I_{j}(k, \tau)+J_{j}(k, \tau)\right. \\
& \left.-(1-2 b) \alpha(N-1)\left\{\frac{v_{j}}{\sqrt{\alpha}}+\phi_{j}(k, \tau)\right\} \delta^{n}(0)\right] \\
G_{i j}(k, t) & \equiv\left[\exp \left\{-K k^{2} t\right\}\right]_{i j}=\exp \left(-k^{2} t\right) K_{i j}+\left(\delta_{i j}-K_{i j}\right)
\end{aligned}
$$

$$
\begin{array}{r}
I_{i}(k, t) \equiv-\sqrt{\alpha} \int \frac{d^{n} p d^{n} q}{(2 \pi)^{n}} \delta^{n}(k-p-q)\left(v_{i} \delta_{l m}+v_{m} \delta_{i l}\right) \phi_{l}(p, t)\left\{-q^{2} \phi_{m}(q, t)+\eta_{m}(q, t)\right\} \\
J_{i}(k, t) \equiv-\alpha \int \frac{d^{n} p d^{n} q d^{n} r}{(2 \pi)^{2 n}} \delta^{n}(k-p-q-r) \phi_{i}(p, t) \phi_{j}(q, t)\left\{-r^{2} \phi_{j}(r, t)+\eta_{j}(r, t)\right\}
\end{array}
$$

After solving the above equation by iteration, we express the result graphically

\section{Fig. 1}

where we denote $\eta$ by a cross or an encircled cross, $G$ a line and $\alpha \delta(0)$ a bullet, respectively. We calculated the one-loop corrections of the two-point function and obtained six $\delta(0)$-divergent diagrams.

\section{Fig. 2}

Each of the six diagrams contributes respectively

$$
\begin{gathered}
2 \alpha N \delta^{n}\left(k+k^{\prime}\right) K_{i j} \frac{1}{\left(k^{2}+k^{\prime 2}\right)^{2}} \int d^{n} p, \\
-4 \alpha N \delta^{n}\left(k+k^{\prime}\right) \theta(0) K_{i j} \frac{1}{\left(k^{2}+k^{\prime 2}\right)^{2}} \int d^{n} p, \\
-13-
\end{gathered}
$$




$$
\begin{gathered}
2 \alpha v^{2} \delta^{n}\left(k+k^{\prime}\right) K_{i j} \frac{1}{\left(k^{2}+k^{\prime 2}\right)^{2}} \int d^{n} p, \\
-4 \alpha v^{2} \delta^{n}\left(k+k^{\prime}\right) K_{i j} \frac{1}{\left(k^{2}+k^{\prime 2}\right)^{2}} \int d^{n} p, \\
4 \alpha v^{2} \delta^{n}\left(k+k^{\prime}\right) \theta(0) K_{i j} \frac{1}{\left(k^{2}+k^{\prime 2}\right)^{2}} \int d^{n} p, \\
-2 \alpha(1-2 b)(N-1)(2 \pi)^{n} \delta^{n}(0) \delta^{n}\left(k+k^{\prime}\right) K_{i j} \frac{1}{\left(k^{2}+k^{\prime 2}\right)^{2}} .
\end{gathered}
$$

The contributions from all the $\delta(0)$-divergent diagrams cancel out if we put $\theta(0)=$ $b \cdot{ }^{[13]}$ It turns out that $\delta^{n}(0)$-divergent term of eq.(2.19) or eq.(4.3) is necessary to the cancellation of the $\delta(0)$ divergences in one-loop order.

Now we apply Langevin equation (3.1) to the model. As discussed in section 3, GCT-covariant and VR-invariant Langevin equation is equivalent to eq.(2.19). We show that the $\delta^{n}(0)$ divergences really cancel out.

With $\Phi_{N}$ substituted by means of constraint (4.2), action (4.1) becomes

$$
\begin{gathered}
S_{N-1}=\int d^{n} x g^{\alpha \beta}(\Phi) \partial_{\mu} \Phi_{\alpha} \partial^{\mu} \Phi_{\beta}, \\
g^{\alpha \beta}(\Phi)=\delta_{\alpha \beta}+\frac{\alpha \Phi_{\alpha} \Phi_{\beta}}{1-\alpha \Phi_{\gamma} \Phi_{\gamma}}, \quad \alpha, \beta, \gamma=1,2, \cdots, N-1 .
\end{gathered}
$$

We can use Langevin equation (3.1) for the system, because the assumption (3.21) is satisfied here as shown in Appendix. As we calculate the one-loop contributions to two-point functions, we need Langevin equation to order $\alpha$, which is

$$
\begin{gathered}
\dot{\Phi}_{\alpha}=\partial^{2} \Phi_{\alpha}+\alpha \Phi_{\alpha} \partial_{\mu} \Phi_{\beta} \partial_{\mu} \Phi_{\beta}-\alpha(N-1) \delta^{n}(0) \Phi_{\alpha}+\alpha b N \delta^{n}(0) \Phi_{\alpha}+\xi_{\alpha}, \\
\xi_{\alpha} \equiv\left(\delta_{\alpha \beta}-\frac{\alpha}{2} \Phi_{\alpha} \Phi_{\beta}\right) \eta_{\beta},
\end{gathered}
$$

where $\eta_{\alpha}$ is white noise. We calculate two-point functions in one-loop order and show that there remains no $\delta^{n}(0)$ divergence. Integral equation corresponding to 
eq. (4.14) is

$$
\begin{array}{r}
\Phi_{\alpha}(x, t)=\int d \tau G(x, t-\tau)\left[\eta_{\alpha}(x, \tau)-\frac{\alpha}{2} \Phi_{\alpha}(x, \tau) \Phi_{\beta}(x, \tau) \eta_{\beta}(x, \tau)\right. \\
\left.+\alpha \Phi_{\alpha}(x, \tau) \partial_{\mu} \Phi_{\beta}(x, \tau) \partial_{\mu} \Phi_{\beta}(x, \tau)+\alpha\{b N-(N-1)\} \delta^{4}(0) \Phi_{\alpha}(x, \tau)\right],
\end{array}
$$

where

$$
G(x, t) \equiv \int \frac{d^{4} k}{(2 \pi)^{4}} \exp (-i k x) \theta(t) \exp \left(-k^{2} t\right)
$$

which can be solved by iteration as eq.(4.8). Eq.(4.16) leads to the following vertices

Fig. 3

In Fig. 3 we denote $\mathrm{G}$ by a line, $\eta_{\alpha}$ a cross or an encircled cross and $\alpha \delta^{n}(0)$ a bullet, respectively. The above vertices contribute to the propagator shown diagramatically in Fig.4.

Fig. 4

In one-loop order the $\delta^{n}(0)$-divergent contributions from Figs. $(4 \mathrm{c}),(4 \mathrm{~d})$ and $(4 \mathrm{e})$ are

$$
\begin{gathered}
\frac{-2 \alpha N}{\left(k^{2}+k^{\prime 2}\right)^{2}} \theta(0) \delta_{\alpha \beta} \delta^{n}\left(k+k^{\prime}\right) \int d^{n} p, \\
\frac{2 \alpha(N-1)}{\left(k^{2}+k^{\prime 2}\right)^{2}} \delta_{\alpha \beta^{\prime}} \delta^{n}\left(k+k^{\prime}\right) \int d^{n} p+2 n d d i v ., \\
\frac{2 \alpha(1-N+b N)}{\left(k^{2}+k^{\prime 2}\right)^{2}}(2 \pi)^{n} \delta_{\alpha \beta} \delta^{n}\left(k+k^{\prime}\right) \delta^{n}(0) .
\end{gathered}
$$

With $\theta(0)=b$, the sum of the above contributions is zero, which coincides with the result of the constrained system. It seems that in $\mathrm{O}(\mathrm{N})$ non-linear $\sigma$ model both Langevin equations (2.19) and (3.1) lead to the correct results. 


\section{5.CONCLUSION}

We constructed Langevin equation (2.19) for constrained system. In the derivation of (2.19), the improved treatment of the stochastic consistency condition for constraints was essential. The Langevin equation can be applied to any system obeying (2.1)-type constraint. From the equation we directly derived Fokker-Planck equation and obtained eq.(2.22) as the equilibrium distribution, which coincides with the one obtained in path integral method. Owing to Ito calculus, the Langevin equation contains $\delta^{n}(0)$-type singular terms and we showed explicitly, in $O(N)$ nonlinear $\sigma$ model, that the singular terms are neccessary to the cancellation of $\delta^{n}(0)$ divergences in one loop order.

Furthermore, we ascertained that eq.(2.19) is equivalent to GCT-covariant and VR-invariant Langevin equation (3.1). We applied eq.(3.1) to $\mathrm{O}(\mathrm{N})$ non-linear $\sigma$ model and showed that the singular terms in GCT-covariant and VR-invariant Langevin equation are also necessary to the cancellation of $\delta^{n}(0)$ divergences in one-loop order.

\section{ACKNOWLEDGMents}

We thank Prof.S.Kawasaki for careful reading of this manuscript and also thank Dr.A.Nakamura for valuable discussion. 


\section{APPENDIX}

Here we show that assumption (3.21) is satisfied in $\mathrm{O}(\mathrm{N})$ non-linear $\sigma$ model. We choose polar coordinates as new variables $Q^{\mu}$ 's in section 3 and immediately recognize that $e_{i}^{A} \nabla_{B} e^{B}{ }_{i}$ vanishes. If the assumption (3.21) is ascertained in the above special case, it is satisfied for any $\left\{Q^{\mu}\right\}$ because of the covariance of the assumption.

We start with a set of variables $\left\{q_{i}\right\}(\mathrm{i}=1,2, \cdots, \mathrm{N})$ obeying $\left(q_{i} q_{i}\right)^{\frac{1}{2}}=r_{c}\left(r_{c}=\right.$ constant) and introduce a new set of variables $\left\{Q^{\mu}\right\}(\mu=1,2, \cdots, N)$. In accordance with section 3

$$
Q^{a} \equiv Q^{N}=\left(q_{i} q_{i}\right)^{\frac{1}{2}}-r_{c}
$$

If we choose $\mathrm{N}-1$ angles of $\mathrm{N}$ dimensional polar coordinates as $Q^{A}$ 's $(A=1,2, \cdots, \mathrm{N}-1)$

$$
\begin{aligned}
q_{1} & =r_{c} \cos Q^{1}, \\
q_{2} & =r_{c} \sin Q^{1} \cos Q^{2}, \\
& \vdots \\
q_{N-1} & =r_{c} \sin Q^{1} \sin Q^{2} \cdots \sin Q^{N-2} \cos Q^{N-1}, \\
q_{N} & =r_{c} \sin Q^{1} \sin Q^{2} \cdots \sin Q^{N-2} \sin Q^{N-1},
\end{aligned}
$$

we obtain the vielbein $e_{i}^{A}$

$$
\begin{aligned}
e_{i}^{A} & \equiv \frac{\partial Q^{A}}{\partial q_{i}}=\frac{\cos Q^{A} \sin Q^{A+1} \cdots \sin Q^{i-1} \cos Q^{i}}{r_{c} \sin Q^{1} \cdots \sin Q^{A-1}}, \quad(i>A, i \neq N), \\
e^{A} & =0, \quad(A>i), \\
e_{i}^{A} & =\frac{-\sin Q^{A}}{r_{c} \sin Q^{1} \cdots \sin Q^{A-1}}, \quad(i=A), \\
e^{A}{ }_{N} & =\frac{\cos Q^{A} \sin Q^{A+1} \cdots \sin Q^{N-1}}{r_{c} \sin Q^{1} \cdots \sin Q^{A-1}},
\end{aligned}
$$

and $e^{a}{ }_{i}$

$$
\begin{aligned}
e_{i}^{a} & \equiv \frac{\partial Q^{N}}{\partial q_{i}}=\sin Q^{1} \cdots \sin Q^{i-1} \cos Q^{i}, \quad(i \neq N), \\
e^{a}{ }_{N} & =\sin Q^{1} \cdots \sin Q^{N-1} .
\end{aligned}
$$


The vielbein satisfies (3.5)

$$
e_{i}^{A} e_{i}^{a}=0
$$

Vielbein $e_{i}^{A}$ leads to metric

$$
g^{A B} \equiv e_{i}^{A} e_{i}^{B}=\frac{1}{r_{c}^{2} \sin ^{2} Q^{1} \cdots \sin ^{2} Q^{A-1}} \delta^{A B} .
$$

By straightforward calculation we obtain

$$
e_{i}^{A} \nabla_{B} e_{i}^{B}=0,
$$

which holds also for other choices of $\left\{Q^{\mu}\right\}$ due to the covariance of the equation. As we proved that assumption (3.21) is satisfied in $\mathrm{O}(\mathrm{N})$ non-linear $\sigma$ model, we are allowed to use the equation (3.1) in section 4. 


\section{REFERENCES}

1. G.Parisi and Y.Wu, Sci. Sin. 24 (1981)483

2. For a review, P.H.Damgaad and H.Hüffel, Phys. Rep. 152 (1987)227

3. M.Namiki, I.Ohba, K.Okana and Y.Yamanaka, Prog. Theor. Phys. 69 (1983) 1580

4. A.Nakamura, Prog. Theor. Phys. 86 (1991)925

5. M.Namiki, I.Ohba and K.Okano, Prog. Theor. Phys. 72 (1984) 350

6. K.Ito, Proc. Imp. Acad. 20 (1944)519

7. R.Graham, Phys. Lett. 109A (1985)209;

R.Mochizuki, Mod. Phys. Lett. A5 (1990)2335;

H.Rumpf, Phys. Rev. D33 (1986)942

8. R.Mochizuki, Prog. Theor. Phys. 85 (1991)407

9. J.Zinn-Justin, Nucl. Phys. B275[FS17] (1986) 135;

N.Nakazawa and D.Ennyu, Nucl. Phys. B305[FS23] (1988) 516

10. L.D.Faddeev,Teoret. i Mat. Piz. 1 (1969)3;

P.Senjanovich, Ann. of Phys. 100 (1976)227;

T.Maskawa and H.Nakajima, Prog. Theor. Phys. 56 (1976)1295

11. L.Arnold, Stochastic Differential Equations (Wiley-Intersciense, New York, 1974)

12. T.L.Stratonovich, Conditional Markov processes and their application to the theory of optimal control (Elsevier, New York, 1968)

13. H.Kawara, M.Namiki, H.Okamoto and S.Tanaka, Prog. Theor. Phys. 84 (1990)749;

N.Komoike, Prog. Theor. Phys. 86 (1991)575 


\section{Figure Captions}

Fig.1 Vertices from eq.(4.8)

Fig.2 $\delta^{n}(0)$-divergent diagrams contributing to two-point function

Fig.3 Vertices from eq.(4.16)

Fig.4 Propagator including vertices shown in Fig.3 